\title{
Cognitive adaptations arising from nonnative experience of sign language in hearing adults
}

\author{
Madeleine Keehner \\ University of Dundee, Dundee, Scotland \\ AND \\ Susan E. Gathercole \\ University of Durham, Durham, England
}

\begin{abstract}
Three experiments examined spatial transformation abilities in hearing people who acquired sign language in early adulthood. The performance of the nonnative hearing signers was compared with that of hearing people with no knowledge of sign language. The two groups were matched for age and gender. Using an adapted Corsi blocks paradigm, the experimental task simulated spatial relations in sign discourse but offered no opportunity for linguistic coding. Experiment 1 showed that the hearing signers performed significantly better than the nonsigners on a task that entailed $180^{\circ}$ rotation, which is the canonical spatial relationship in sign language discourse. Experiment 2 found that the signers did not show the typical costs associated with processing rotated stimuli, and Experiment 3 ruled out the possibility that their advantage relied on seen hand movements. We conclude that sign language experience, even when acquired in adulthood by hearing people, can give rise to adaptations in cognitive processes associated with the manipulation of visuospatial information.
\end{abstract}

Sign language raises some interesting questions for working memory theory. Traditional conceptualizations of working memory postulate separable, modality specific subsystems for processing visuospatial and verbal information (Baddeley, 1986; Baddeley \& Hitch, 1974). A sound-based phonological code is assumed to underlie the processing of linguistic stimuli, whether auditory (heard speech) or phonologically recodable visual inputs, such as print or lip-read stimuli (Campbell \& Dodd, 1980; Gardiner, Gathercole, \& Gregg, 1983). Sign language, however, is a visuospatial linguistic form that uses observed movements in space to transmit information. The fact that sign and speech exist within separate sensory domains raises the following important theoretical question: Does signing experience produce adaptations to working memory functions?

One domain in which such adaptations have been found is that of verbal working memory. Evidence from deaf native signers suggests that cognitive mechanisms analogous to those for speech permit the internal representation of sign movements in a code that preserves their sensory or physical properties. Formationally similar signs produce confusion errors in immediate memory tasks (Poizner, Bellugi, \& Tweney, 1981; Wilson \& Emmorey, 1997), suggesting that handshapes are preserved in the memory representation. Recall errors for signs are not related to the phonological or orthographic forms of corresponding words, but are visually or kinesthetically very similar to the target signs, implying that sign movements are maintained in short-term memory (Klima, Tzeng, Fok, Bellugi, \& Corina, 1996). A sign length effect has been reported, with smaller recall spans for long signs than for short signs, indicating an articulatory sign-rehearsal process that is time limited (Wilson \& Emmorey, 1998) and closely analogous to articulatory rehearsal of speech (Baddeley, Thomson, \& Buchanan, 1975). Concurrent irrelevant hand movements reduce recall of signs in deaf native signers (Wilson \& Emmorey, 1997), a process analogous to the articulatory suppression effect, which is assumed to interfere with internal rehearsal processes for words (Baddeley et al., 1975). These findings provide compelling support for an "inner voice of the hands" (Klima \& Bellugi, 1979), an internal representation that preserves the physical or formational properties of sign movements, just as a phonological code preserves the sensory properties of speech sounds, and that permits the rehearsal of signs in working memory via a kinesthetic or motor articulatory code (Wilson \& Emmorey, 1997).

Another domain in which adaptations from signlanguage experience have been found is visuospatial working memory. Spatial representations are uniquely important to sign language. Sign movements are produced within signing space, a 3-D area located in front of the signer's body, extending to the waist, the top of the head and the lateral reach of the forearms. Topographical spatial relations can be mapped in signing space (Emmorey,

M. Keehner, m.m.keehner@dundee.ac.uk 
Corina, \& Bellugi, 1995). Classifier signs (handshapes that represent a class of objects) can be manipulated to show the real-world locations, orientations, and movements of objects. Signing space can also be used in a more abstract manner to specify syntactical or grammatical relationships among referents. Unlike speech sounds, different components of signs can be produced concurrently, so that grammatical constructions can be transmitted in parallel, with location and spatial organization fulfilling the syntactic functions achieved by serial word order in speech. Signers routinely generate and manipulate visuospatial representations. For example, because British Sign Language (BSL) has no signs to indicate the pronouns he or she, a BSL signer describing an event involving a number of people identifies the relevant individuals, assigns each one to a locus in signing space, and then refers to each referent periodically by pointing to or looking at its hypothetical location. Both signer and addressee must generate and maintain these imagined spatial locations for the duration of the discourse.

These unique linguistic properties suggest that spatial representations have a special importance in sign language, a claim supported by neurophysiological research. Unique right hemisphere activations have been found during the processing of sign language, suggesting that space is processed concomitantly with linguistic features and has an integral role within the message (Bavelier et al., 1997; Corina, 1998; Hickok, Bellugi, \& Klima, 1996; Söderfeldt, Rönnberg, \& Risberg, 1994).

Given the need to process rapidly fluctuating movements in space, native signers might be expected to outperform nonsigners on tasks involving dynamic spatial information. Deaf signers are better than hearing nonsigners at identifying the direction of movement in peripheral vision (Neville \& Lawson, 1987), and deaf signing children are better than hearing children at analyzing and recalling dynamic point light displays such as Japanese kanji figures (Klima et al., 1996; Poizner, Fok, \& Bellugi, 1989). Deaf signers outperform nonsigners on tasks such as scanning visual stimuli (Rettenbach, Diller, \& Sireteanu, 1999) and rapidly shifting visual attention from one spatial location to another (Parasnis \& Samar, 1985). Deaf children have enhanced memory for spatial locations, orientation, and movement, as shown by their superiority to hearing agematched controls on the Corsi spatial-span task (Wilson, Bettger, Niculae, \& Klima, 1997). Native signers perform more accurately than nonsigners on image generation tasks (Emmorey, Kosslyn, \& Bellugi, 1993), on detection of mirror-image reversals (Emmorey et al., 1993), and on mental rotation tasks, especially those that involve $180^{\circ}$ rotation in the horizontal plane (Emmorey, Klima, \& Hickok, 1998).

Taken together, these findings indicate that native signing experience produces adaptations to both verbal and visuospatial working memory functions. Native signers develop the ability to represent internally the movements of sign language and accrue enhancements to visuospatial functions. The fact that hearing people exposed to sign language from birth have shown many of these effects, whereas deaf nonsigners generally perform at the same levels as do hearing nonsigners (Emmorey et al., 1993), implies that experience with a visuospatial language, rather than lack of auditory input, is responsible for these differences. Since research to date has focused on native or nearnative signers, how much signing experience is required to produce these kinds of adaptations, or whether they can accrue from relatively little exposure to the language, is not known. The purpose of the present study was to examine whether nonnative hearing signers have any advantage for cognitive functions related to sign language.

To address this issue, immediate memory performance was assessed, using a type of spatial transformation commonly found in sign language. In face-to-face sign discourse, two individuals are typically positioned roughly opposite each other so that each views the other's signing space as $180^{\circ}$ rotated. In BSL and other sign languages, topographical descriptions are routinely produced from the perspective of the signer rather than from that of the addressee, requiring the addressee who wants to comprehend the spatial relations from the signer's perspective to perform something akin to a spatial transformation (Emmorey et al., 1998). This may explain why native signers show superior performance on tasks that involve mental rotation in the horizontal plane, an advantage seen with both arrays of objects and observed body movements (Emmorey et al., 1998).

In a series of experiments, we examined immediate memory for spatial sequences requiring rotation in two groups of hearing people, signers and nonsigners. The signers had relatively limited signing experience, acquired in early adulthood. In Experiment 1, participants watched a series of spatial movements produced on an array, and attempted to reproduce the movements on a corresponding array rotated by $180^{\circ}$. Like signing, the test entailed dynamic hand movements to positions in 3-D space, but the movements, unlike those used in signing, had no linguistic content. If the signers performed significantly better on this task than the nonsigners, it would indicate that adaptations in the visuospatial domain that generalize beyond sign language arise from relatively limited signing experience in hearing people.

\section{EXPERIMENT 1}

\section{Method}

Participants. In all experiments reported in this paper, two groups of participants were compared, signers and nonsigners. The signers were 12 hearing adults who were competent users of BSL and were working as BSL interpreters. All had acquired BSL as adults, and had 1 to 5 years' regular signing experience (selfreported as daily or almost daily). All of the signers had undertaken a BSL interpreter training course at the Centre for Deaf Studies at the University of Bristol (one participant was completing her training at the time of testing), either as a 2- or 3-year undergraduate program, or as a 2-year postgraduate diploma following a bachelor's degree in a different discipline. The nonsigners were 12 hearing adults with no knowledge of BSL ( 10 of the 12 were postgraduate students at the University of Bristol). The two groups were matched for gender (9 females, 3 males per group) and age (signers' age range, 2334 years, mean age, 27.4 years; nonsigners' age range, $21-35$ years, mean age, 27.1 years). Mean years of full time education were as follows: signers, 16.2 years ( $S D=1.47$; range, $14-18$ years); nonsigners, 17.1 years $(S D=1.83$; range, $12-19$ years $)$. 
Spatial span. Prior to commencing the experiments reported here, the two groups were compared on the Corsi blocks spatial-span test. This task is widely used in experimental research and neuropsychological testing as a measure of spatial short-term memory (STM) capacity (Corsi, 1973; Hanley, Young, \& Pearson, 1991; Hitch, Halliday, Dodd, \& Littler, 1989; Milner, 1971). It is considered somewhat analogous to digit span as a measure of visuospatial STM, and has been shown to load onto a passive visuospatial storage factor (Richardson \& Vecchi, 2002). In this task, the experimenter taps a spatial sequence on a quasirandom array of blocks, and the participant immediately attempts to tap the same spatial sequence, preserving the order. Sequences of three, four, five, six, seven, and eight items were presented, using a standard span procedure. Mean scores (and SDs) for signers and nonsigners were 14.50 (4.32) and 13.08 (3.53), respectively. The range of scores was identical for the two groups (for both signers and nonsigners, where the maximum possible score was 24 , the group minimum was 8 , and the group maximum was 20). An independent samples $t$ test found no significant difference $[t(22)=$ $.88, p=.39, C I_{95}=-4.75$ to 1.92$]$, indicating that the signers and nonsigners did not differ in spatial STM span.

Design and Materials. A task was devised as a nonverbal analogue of spatial relations in sign language. When two signers face each other, each views the other's signing space as $180^{\circ}$ rotated. The spatial layout of the test materials was designed to model this relationship. Two identical sets of Corsi blocks (Milner, 1971) were constructed. Each set comprised a wooden board $(350 \mathrm{~mm} \times 250 \mathrm{~mm})$ with wooden cubes attached in a quasirandom pattern. The configuration of the blocks on each board was identical. Both sets were painted black to remove potential color cues. The experimenter and participant were seated facing each other across a table. One Corsi set (set A) was placed on the table top immediately in front of the experimenter, and the second set (set B) was positioned directly opposite, immediately in front of the participant. Each set of blocks was rotated $180^{\circ}$ relative to the orientation of the other set, so that each array faced the person in front of it (see Figure 1). Numbers visible only to the experimenter indicated corresponding blocks on the two sets. The stimulus pool comprised random number sets produced by sampling without replacement from digits one to nine. From these, six lists were devised at each length of one, two, three, four, and five items.

Procedure. A familiarization period was given prior to testing. The identical but rotated nature of the two Corsi sets was explained to the participant, and the experimenter demonstrated the correspondence between the configuration of blocks on set A (as viewed from the experimenter's perspective) and set B (as seen from the participant's perspective). Verbal instructions were followed by four one-item practice trials. The experimenter corrected any errors during the practice trials.

Following the practice trials, testing began. Using the index finger of the right hand, the experimenter tapped a spatial sequence on Corsi set $\mathrm{A}$ at a rate of about $1 \mathrm{block} / \mathrm{sec}$. At the end of the sequence a verbal signal was given, and the participant was then required to tap the corresponding $\left(180^{\circ}\right.$ rotated) sequence on Corsi set B, preserving the correct order. The tapping sequences were blocked, with blocks consisting of six trials at each length. Testing began with a list length of one item. After each successive block, list length was increased by one item, ending with a final block at the maximum length of five items. Performance was scored live, using a strict serial recall criterion. A positive score was given for each correct block tapped in the correct serial position in a sequence.

\section{Results}

To allow comparison across different list lengths, serial scores were converted to proportion correctly recalled, calculated as a fraction of the maximum possible score at each list length. All analyses used a minimum alpha level of .05 , with corrected familywise error rates to control Type I errors where necessary, and $95 \%$ confidence intervals $\left(C_{.95}\right)$ for means or differences between means. Assumptions of sphericity were confirmed by Mauchly's test.

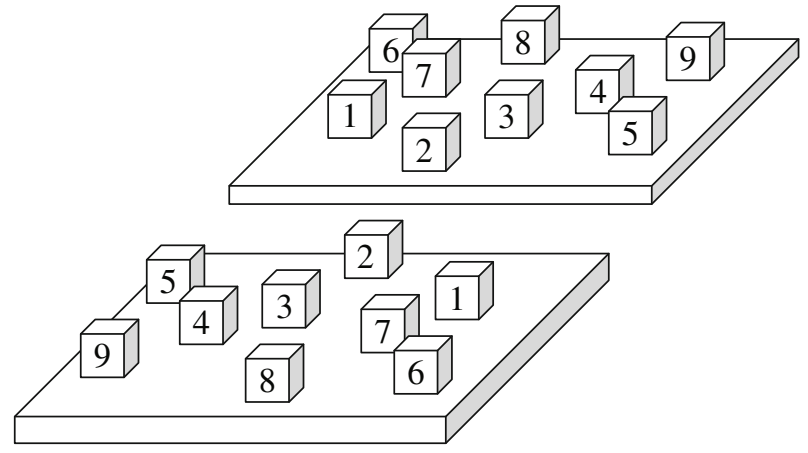

Figure 1. Stimulus setup in Experiment 1, also used in Experiments 2 and 3. Each set of blocks was rotated $180^{\circ}$ relative to the other. The numbers representing corresponding blocks were visible only to the experimenter.

A two-factor $(2 \times 5)$ mixed design ANOVA was performed on proportion correct, with group (signers, nonsigners) as a between-subjects factor and list length (one, two, three, four, and five items) as a within-subjects factor. This found a significant main effect of group $[F(1,22)=$ $\left.11.30, p=.003, \eta_{\mathrm{p}}^{2}=.34\right]$. The main effect of list length was also significant $\left[F(4,88)=38.573, p<.001, \eta_{\mathrm{p}}^{2}=\right.$ 89], but there was no interaction between group and list length $\left[F(4,88)=1.07, p=.38, \eta_{\mathrm{p}}^{2}=.05\right]$. Figure 2 shows proportions correct achieved by signers and nonsigners at each list length.

Serial position data from the five-item lists for each group are summarized in Figure 3. Both groups showed a strong primacy effect. Signers and nonsigners achieved comparable recall rates up to the second item, but the nonsigners' scores rapidly declined after this point.

Error rates were compared, using independent samples $t$ tests. Two types of errors were scored for each individual: (1) item errors, in which the participant failed entirely to recall a target item; and (2) order errors, in which the participant recalled a target item but in the wrong serial position. Nonsigners made significantly more item errors than signers $[t(22)=2.86, p=.009]$, but the two groups

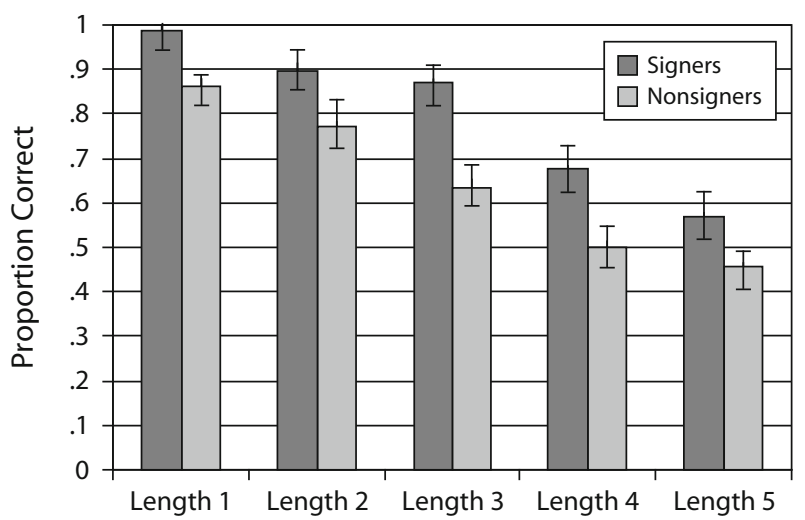

Figure 2. Recall performance (proportion correct) of signers and nonsigners in Experiment 1, by list length. Error bars represent standard error. 


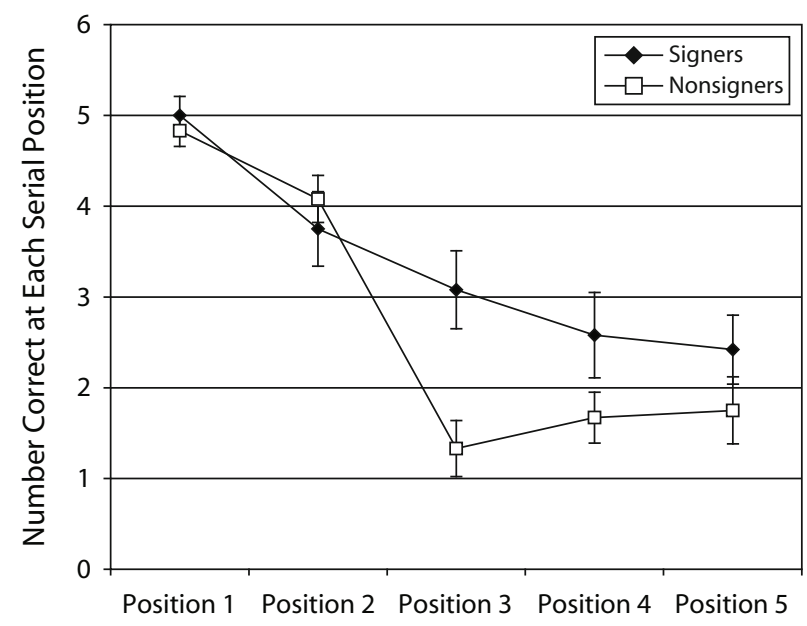

Figure 3. Serial position data for signers and nonsigners on the five-item lists in Experiment 1. Data points represent total number recalled at each serial position (maximum possible $=6$ ). Error bars represent standard error.

did not differ significantly in the number of order errors made $[t(22)=1.89, p=.07]($ see Table 1$)$.

\section{Discussion}

On this task, signers performed significantly better than nonsigners, with significantly higher serial recall scores resulting from greater levels of accuracy in item but not order. The serial position data showed that the two groups performed similarly on initial items in a sequence, but nonsigners' recall declined more sharply on subsequent list positions.

This novel finding suggests that nonnative experience with sign language has enhanced the signers' ability to encode, transform, and reproduce sequences of movements on a spatial array rotated by $180^{\circ}$. The experimental task mimicked spatial relations in face-to-face sign discourse, but the movements presented were not sign movements so they carried no potential for linguistic encoding.

A key theoretical question raised by this finding is which cognitive mechanisms have become enhanced through signing experience. A likely candidate is visuospatial working memory (Baddeley, 1986; Logie, 1995). One possibility is that the signers have acquired an advantage for maintaining information in visuospatial shortterm memory (STM). Signers routinely have to remember the positions and relations of real or imagined loci in space during sign discourse. If such processes had accrued ben-

Table 1

Total Number of Item Errors and Order Errors (Averaged Across Participants) Made by Signers and Nonsigners in Experiment 1

\begin{tabular}{lccccc}
\hline & \multicolumn{2}{c}{ Item Errors } & & \multicolumn{2}{c}{ Order Errors } \\
\cline { 2 - 3 } \cline { 5 - 6 } & Total & $S D$ & & Total & $S D$ \\
\hline Nonsigners* & 23.83 & 8.22 & & 13.67 & 9.19 \\
Signers & 14.58 & 4.19 & & 10.67 & 5.85 \\
\hline
\end{tabular}

${ }^{*} p<.05$. efits to visuospatial STM capacity, the signers might have been able to more readily maintain the original sequence, potentially freeing up cognitive resources for performing the transformation. This event could also have accounted for the serial position data, which showed a less sharp decay on later items for the signers. However, the data from the Corsi blocks spatial-span test, administered prior to Experiment 1, do not support this account. This measure indicated no difference between the groups in spatial STM capacity, suggesting that the signers' advantage lay in their ability to manipulate the spatial information rather than in their ability to merely maintain it.

The results of Experiment 1 raise some questions for further investigation. Previous studies have shown that deaf native signers do not show typical performance costs associated with the requirement to mentally rotate information (Shepard \& Cooper, 1982). Under certain circumstances they perform as well (and sometimes better) with rotated inputs as they do with nonrotated inputs, presumably because "the advantage for processing the canonical (most frequent) linguistic expression overrides the difficulty imposed by mental rotation" (Emmorey et al., 1998, p. 226). The purpose of Experiment 2 was to directly compare rotated and nonrotated inputs within the same experimental paradigm, to establish whether these nonnative hearing signers, like deaf native signers, showed any cost of rotation.

The paradigm in Experiment 2 was similar to that of Experiment 1, but it used three different angles of rotation: $0^{\circ}, 90^{\circ}$, and $180^{\circ}$. Most previous studies have compared $0^{\circ}$ and $180^{\circ}$ only, as the latter is generally acknowledged to be the canonical form in sign discourse. However, signing situations such as conversing in groups give rise to a variety of spatial relations, requiring signers to encode others' signing space from a range of orientations. Given that face-to-face signing relations are typical but not exclusive, we chose to compare both $180^{\circ}$ and $90^{\circ}$ rotations with a $0^{\circ}$, or no rotation, condition.

Experiment 2 allowed us to examine how well signers and nonsigners deal with differently rotated arrays relative to a nonrotated array. If these nonnative signers had accrued similar effects to those previously found in native signers, their performance on rotated trials should not differ significantly from their performance on nonrotated trials. By contrast, we expected nonsigners to show the typical cost of mental rotation; that is, their performance on the rotated arrays should be significantly lower than their performance on the nonrotated array.

\section{EXPERIMENT 2}

\section{Method}

Participants. The same two groups of participants took part as in Experiment 1.

Design and Materials. As in Experiment 1, two identical Corsi sets were used. Each set consisted of a wooden board with numbered wooden cubes attached in a quasirandom pattern. The configuration of the blocks on each board was identical, and both sets were painted black to remove potential color cues. The experimenter and participant were seated on opposite sides of a table facing each other. One Corsi set (set A) was placed on the table top immediately in front of the experimenter, and the second set (set B) was positioned directly 
opposite, immediately in front of the participant. Three different orientation conditions were presented: $0^{\circ}, 90^{\circ}$, and $180^{\circ}$. In the $0^{\circ}$ condition, the orientations of both sets were identical (set B was not rotated relative to set A; see Figure 4). In the $90^{\circ}$ orientation condition, set B was rotated $90^{\circ}$ relative to set A (see Figure 5). In the $180^{\circ}$ orientation condition, set $\mathrm{B}$ was rotated $180^{\circ}$ relative to set A (this condition was identical to the setup in Experiment 1; see Figure 1). Numbers visible only to the experimenter indicated corresponding blocks on the two sets. The stimulus pool comprised random number sets produced by sampling without replacement from digits one to nine. From these, four lists were devised at each length of one, two, three, four, and five items. The stimulus sets and the testing order of conditions were counterbalanced using a Latin square design.

Procedure. Prior to testing in each orientation condition, a familiarization period was given. The relationship between the two Corsi sets was explained to the participant, and the experimenter demonstrated the correspondence between the configuration of blocks on set A and set B. Verbal instructions were followed by four one-item practice trials. The experimenter corrected any errors during the practice trials. Following the practice trials, testing began. The experimenter tapped a spatial sequence on Corsi set $\mathrm{A}$ at a rate of about $1 \mathrm{block} / \mathrm{sec}$. At the end of the sequence a verbal signal was given, and the participant was then required to tap the corresponding sequence on Corsi set B, preserving the correct order and taking account of the angle of rotation, if any. The tapping sequences were blocked, with blocks consisting of four trials at each length. Testing began with a list length of one. After each successive block, list length was increased by one item, ending with a final block at the maximum length of five items. This general procedure was followed for all three orientation conditions. To assess whether ceiling effects might be masking a difference in the less demanding $0^{\circ}$ condition, additional sets of 6 and 7 items in length were administered for this orientation only. Performance was scored live, using a strict serial recall criterion. A positive score was given for each correct block tapped in the correct serial position in a sequence.

\section{Results}

Figure 6 shows proportion correct for signers and nonsigners at all list lengths in the three orientation conditions $\left(0^{\circ}, 90^{\circ}\right.$, and $\left.180^{\circ}\right)$. The pattern of data indicates that the signers are predominantly scoring above the nonsigners in the rotated conditions $\left(90^{\circ}\right.$ and $\left.180^{\circ}\right)$, but not in the nonrotated $\left(0^{\circ}\right)$ condition.

To lessen the impact of ceiling effects, we excluded from the analyses all one-item trials (in the $0^{\circ}$ condition, ceiling effects are also evident at list lengths 2 and 3, but

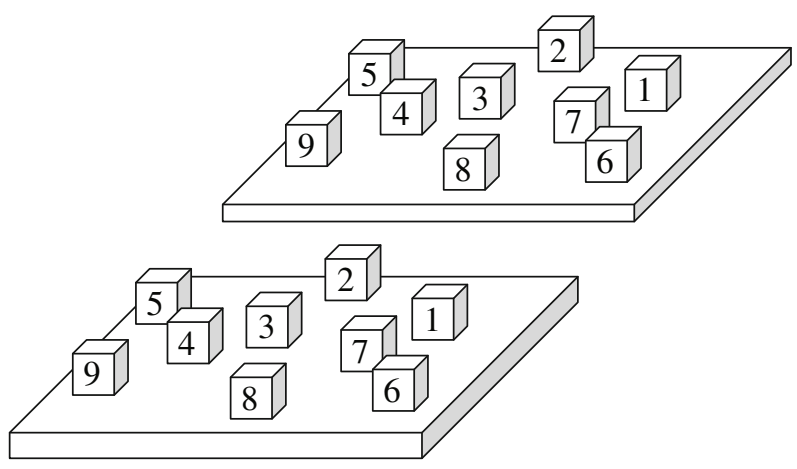

Figure 4. Zero-degree rotation stimulus setup used in Experiments 2 and 3 . The blocks were not rotated relative to each other (no rotation or $0^{\circ}$ condition). The numbers representing corresponding blocks were visible only to the experimenter.

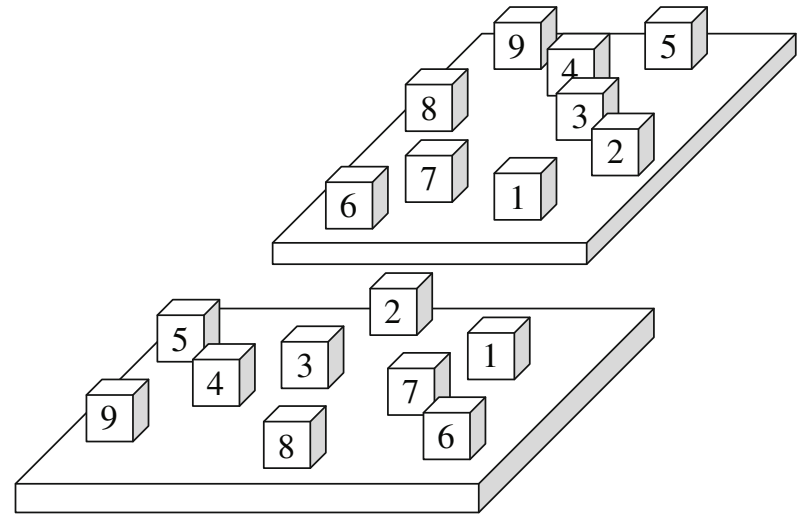

Figure 5. Ninety-degree rotation stimulus setup used in Experiments 2 and 3. Each set of blocks was rotated $90^{\circ}$ relative to the other. The numbers representing corresponding blocks were visible only to the experimenter.

we did not exclude those list lengths, because in the more demanding rotated conditions this would have had a disproportionate impact and could potentially have distorted the pattern of results). Figure 7 shows proportion correct collapsed across list lengths 2 to 5. Nonsigners show a typical pattern, with performance declining sharply between the $0^{\circ}$ condition and the rotation conditions of $90^{\circ}$ and $180^{\circ}$. For signers, the decline in performance is less steep.

A two-factor $(2 \times 3)$ mixed design ANOVA was performed on proportion correct, with group (signers, nonsigners) as a between-subjects factor and angle $\left(0^{\circ}\right.$, $90^{\circ}$, and $180^{\circ}$ ) as a within-subjects factor. There was a significant main effect of angle $[F(2,44)=24.06, p<$ $\left..001, \eta_{\mathrm{p}}^{2}=.52\right]$, reflecting the fact that rotated arrays were more difficult overall than nonrotated arrays. There was no main effect of group $\left[F(1,22)=.32, p=.42, \eta_{\mathrm{p}}^{2}=\right.$ $.02]$, but there was a significant interaction of group by angle $\left[F(2,44)=5.31, p=.009, \eta_{\mathrm{p}}^{2}=.19\right]$, suggesting that signers and nonsigners were differently affected by rotation.

This interaction term was explored using a simple effects analysis with Bonferroni familywise alpha adjustment. Nonsigners showed the typical cost of mental rotation. Their scores at $0^{\circ}$ were significantly higher than their scores at $90^{\circ}\left(p<.001, C I_{95}=.10\right.$ to .36$)$, and significantly higher than their scores at $180^{\circ}\left(p<.001, C I_{.95}=\right.$ .21 to .47$)$. In contrast, signers' scores at $0^{\circ}$ were not significantly different from their scores at $90^{\circ}\left(p=.44, C I_{.95}=\right.$ -.05 to .20$)$ or from their scores at $180^{\circ}\left(p=.06, C I_{95}=\right.$ -.01 to .25). The simple effects of angle were significant for nonsigners $\left[F(2,21)=22.49, p<.001, \eta_{\mathrm{p}}^{2}=.68\right]$ but not for signers $\left[F(2,21)=3.00, p=.07, \eta_{\mathrm{p}}^{2}=.22\right]$.

The difference between signers and nonsigners did not reach significance at the adjusted alpha level in any of the three conditions, which may have been an artifact of this paradigm or may have resulted from a lack of power, but the trends in the data for the $180^{\circ}$ condition were consistent with Experiment 1 . To ensure that a real difference between the groups in the less demanding $0^{\circ}$ condition was not being masked by ceiling effects, scores from lon- 
ger sets at this orientation were also compared. For consistency with typical spatial span procedure, we compared proportion correct collapsed across sets of 3 to 7 items. The means (and $S D \mathrm{~s}$ ) for signers and nonsigners were .77 (.20) and $.76(.08)$, respectively. An independent samples $t$ test confirmed that there was no significant difference between signers and nonsigners in this condition $[t(22)=$ $.14, p=.89, C I_{.95}=-.12$ to .14$]$.

\section{Discussion}

In Experiment 2, the interaction and simple effects analyses indicate that signers and nonsigners were differently affected by the three orientation conditions. The nonsigners showed a pattern of data typical for rotated arrays, in which performance was best when no rotation was required and deteriorated significantly when rotation was required. By contrast, the signers showed no significant difference in performance between $0^{\circ}$ and $90^{\circ}$, or between $0^{\circ}$ and $180^{\circ}$. This attenuation of the typical mental rotation effect concurs with previous findings from deaf native signers (Emmorey et al., 1998). It suggests that these hearing individuals have accrued an advantage for processing arrays at orientations that differ from their own, consistent with the demands of sign language.

This question remains: What kind of process underpins the signers' advantage for reproducing rotated arrays? One possible candidate is a spatial transformation process, such as mental rotation of the array, or an imagined perspective shift. It is possible that signing experience, in which one must routinely process the signing space of others at a range of orientations, leads to enhancement of spatial transformation abilities. This account could explain the results of Experiments 1 and 2; an enhanced ability for this type of process could produce a significant advantage for the most commonly encountered type of rotation $\left(180^{\circ}\right)$, and it would presumably attenuate typical rotation costs even for less common orientations, such as $90^{\circ}$.

A possible alternative account is that the signers encoded and reproduced the experimenter's movements, rather than the spatial locations of the target blocks. As we discussed earlier, much evidence indicates that native signers represent sign movements internally, using a kinesthetic or motor code. Some investigators have postulated that signers perceive signed descriptions as if they were producing the input themselves. Under this motor theory of sign perception, "signers perform a transformation of the perceived articulation into a reversed representation of their own production" (Emmorey et al., 1998, p. 241). In support of this theory, Emmorey et al. (1998) showed that deaf native signers incurred no cost of rotation only when the to-be-rotated arrays were presented as movements executed in signing space; when sequences of objects were presented, a rotation cost was evident (although deaf native signers still performed better than nonsigners). If signing experience enhances the representation of motor patterns, the signers in the present study might have been able to encode the hand movements of the experimenter seated opposite. If these movements were then replicated in egocentric coordinates, it would provide the correct pattern of tapping on a $180^{\circ}$ rotated array. By adjusting their imagined head-

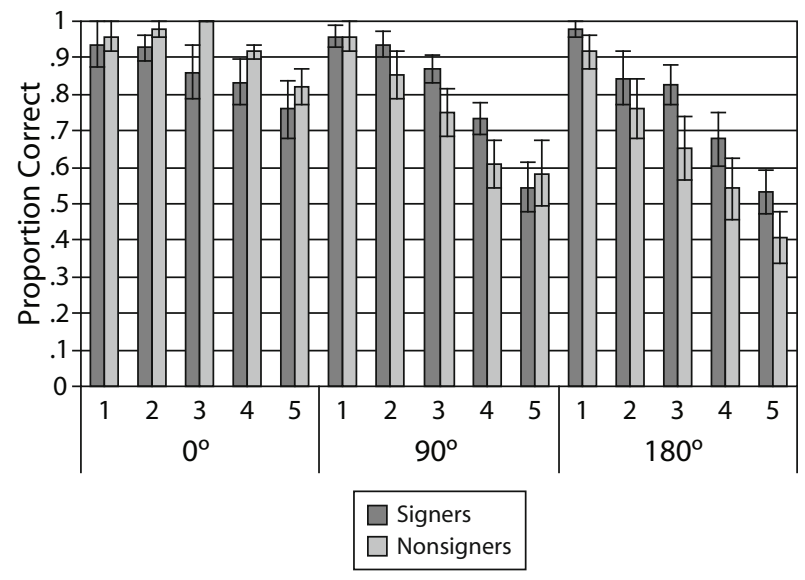

Figure 6. Recall performance (proportion correct) of signers and nonsigners in the three orientation conditions in Experiment 2, by list length. Error bars represent standard error.

ing to align with the array, it would also be possible to apply this process to a $90^{\circ}$ rotated array. This could potentially explain the signers' advantage at $180^{\circ}$ in Experiment 1 , and their attenuated rotation costs in Experiment 2.

If the signers depend on seen hand movements to engage such a motor representation, it should become evident if the sequences are presented without hand movements. With no motor pattern to encode, the signers should lose their advantage. Experiment 3 explored this hypothesis by presenting analogous spatial sequences to those in Experiment 2, but with no associated hand or arm movements by the experimenter. In Experiment 3, the sequences were presented via small lights mounted on the blocks in the array. If the process does not rely on observed motor patterns, the data should look the same even when the sequences are presented without body movements; that is, the signers will retain their relative advantage for processing $180^{\circ}$ rotated arrays, and a comparison of rotated ver-

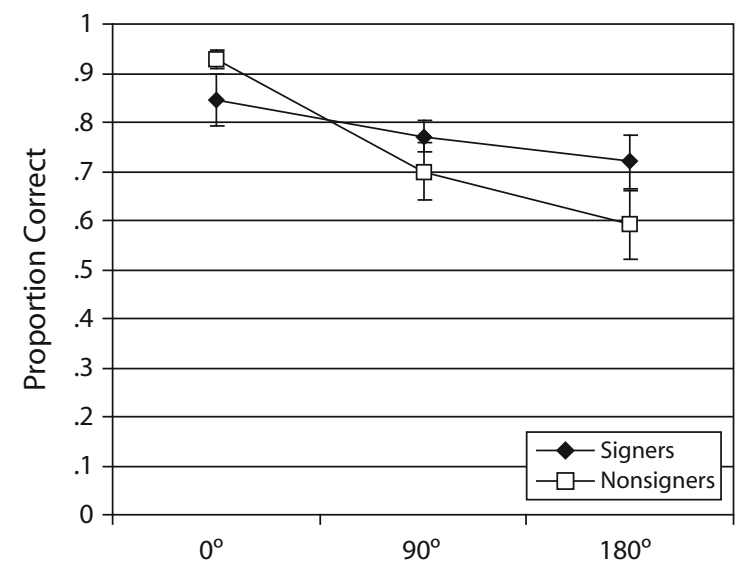

Figure 7. Recall performance (proportion correct) of signers and nonsigners in the three orientation conditions in Experiment 2, collapsed across list lengths 2 to 5 . Error bars represent standard error. 


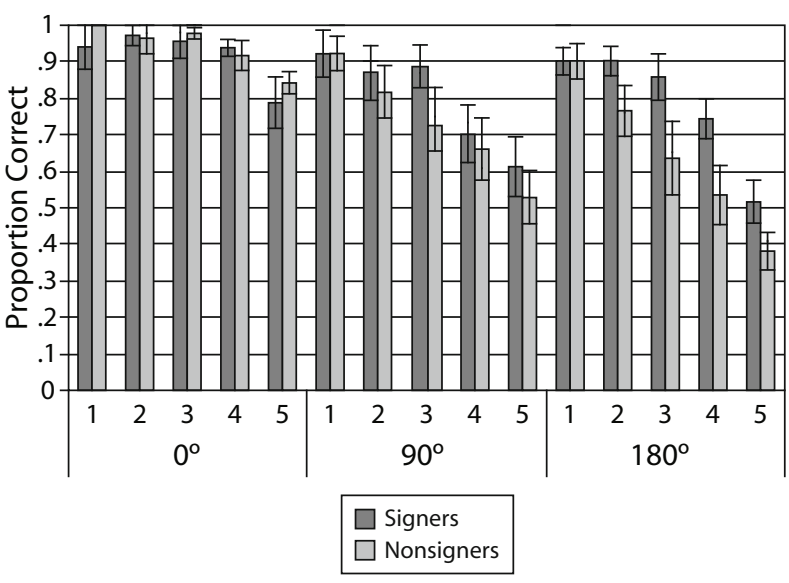

Figure 8. Recall performance (proportion correct) of signers and nonsigners in the three orientation conditions in Experiment 3, by list length. Error bars represent standard error.

sus nonrotated conditions will show that the typical cost of rotation is attenuated among signers only.

\section{EXPERIMENT 3}

\section{Method}

Participants. The same two groups of participants were compared as in Experiments 1 and 2.

Design and Materials. As in Experiments 1 and 2, two identical Corsi sets were used. Each set consisted of a wooden board with numbered wooden cubes attached in a quasirandom pattern. On the Corsi set closest to the experimenter (set A), a light-emitting diode (LED) was attached to the top surface of each block. The LEDs were operated remotely by means of a small handheld keypad constructed with momentary switches, which allowed brief illumination of the LEDs at each location. Each number on the keypad operated the LED on the corresponding numbered block. The configuration of the blocks on each board was identical, and both sets were painted black to remove potential color cues. The experimenter and participant were seated on opposite sides of a table facing each other. One Corsi set (set A) was placed on the table top immediately in front of the experimenter, and the second set (set B) was positioned directly opposite, immediately in front of the participant. As in Experiment 2, three different orientation conditions were presented: $0^{\circ}, 90^{\circ}$, and $180^{\circ}$. In the $0^{\circ}$ condition, the orientations of both sets were identical (set B was not rotated relative to set A; see Figure 4). In the $90^{\circ}$ orientation condition, set $\mathrm{B}$ was rotated $90^{\circ}$ relative to set A (see Figure 5). In the $180^{\circ}$ orientation condition, set B was rotated $180^{\circ}$ relative to set A (see Figure 1). Numbers that were visible only to the experimenter indicated corresponding blocks on the two sets. The stimulus pool comprised random number sets produced by sampling without replacement from digits one to nine. From these, four lists were devised at each length of one, two, three, four, and five items. The stimulus sets and order of conditions were counterbalanced using a Latin square design.

Procedure. Prior to testing in each orientation condition, a familiarization period was given. The relation between the two Corsi sets was explained to the participant, and the experimenter demonstrated the correspondence between the configuration of blocks on set A and set B. Verbal instructions were followed by four one-item practice trials during which any errors were corrected. Following the practice trials, testing began. Using the remote keypad, held out of view of the participant, the experimenter illuminated a sequence of LEDs on Corsi set A, at a rate of about one per second. At the end of the sequence, a verbal signal was given, and the participant was then required to tap the corresponding sequence on Corsi set $\mathrm{B}$, preserving the correct order and taking account of the angle of rotation, if any. The LED sequences were blocked, with blocks consisting of four trials at each length. Testing began with a list length of one. After each successive block, list length was increased by one item, ending with the maximum length of five items. This general procedure was followed for all three orientation conditions. To assess whether ceiling effects might be masking a difference in the less demanding $0^{\circ}$ condition, additional sets of 6 and 7 items in length were administered for this orientation only. Performance was scored live, using a strict serial recall criterion. A positive score was given for each correct block tapped in the correct serial position in a sequence.

\section{Results}

Figure 8 shows proportion correct at each list length for signers and nonsigners, with stimuli presented via LEDs at the three angles $\left(0^{\circ}, 90^{\circ}\right.$, and $\left.180^{\circ}\right)$. The patterns of data are consistent with Experiment 2: The signers are for the most part scoring above the nonsigners in the rotated conditions $\left(90^{\circ}\right.$ and $\left.180^{\circ}\right)$ but not in the nonrotated $\left(0^{\circ}\right)$ condition.

To lessen the impact of ceiling effects, we excluded from further analysis all one-item trials (as in the previous experiment, ceiling effects are also evident at list lengths 2 and 3 in the $0^{\circ}$ condition, but we did not exclude those list lengths because in the rotated conditions this would have a disproportionate impact, potentially distorting the pattern of results). Figure 9 shows proportion correct collapsed across list lengths 2 to 5. The pattern of findings is similar to that for Experiment 2; that is, nonsigners show a typical pattern, with performance considerably lower in the rotation conditions compared with no rotation, whereas for signers the difference in performance between rotation and no rotation is less marked.

A two-factor $(2 \times 3)$ mixed design ANOVA was performed on proportion correct, with group (signers, nonsigners) as a between-subjects factor and angle $\left(0^{\circ}, 90^{\circ}\right.$, and $180^{\circ}$ ) as a within-subjects factor. There was a significant main effect of angle $[F(2,44)=20.41, p<.001$, $\left.\eta_{\mathrm{p}}^{2}=.48\right]$, indicating generally greater task difficulty under rotation than under no rotation. There was no main effect of group $\left[F(1,22)=2.06, p=.17, \eta_{\mathrm{p}}^{2}=.09\right]$. In

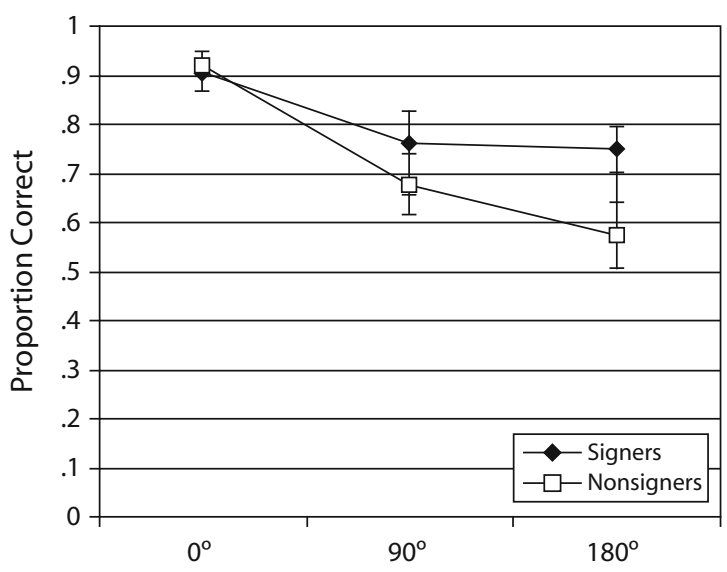

Figure 9. Recall performance (proportion correct) of signers and nonsigners in the three orientation conditions in Experiment 3, collapsed across list lengths 2 to 5. Error bars represent standard error. 
contrast to Experiment 2, there was no significant interaction of group by angle $\left[F(2,44)=2.54, p=.09, \eta_{\mathrm{p}}^{2}=\right.$ .10]. Although the lack of an interaction would normally preclude simple effects analyses, we established a priori the need for pairwise comparisons to assess cost of rotation for the two groups separately. These analyses showed the same general pattern as in Experiment 2. Nonsigners showed the typical cost of mental rotation. Their scores at $0^{\circ}$ were significantly higher than their scores at $90^{\circ}(p=$ $.004, C I_{.95}=.07$ to .41$)$ and significantly higher than their scores at $180^{\circ}\left(p<.001, C I_{.95}=.18\right.$ to .51$)$. In contrast, signers' scores at $0^{\circ}$ did not differ significantly from their scores at $90^{\circ}$ ( $p=.11, C I_{.95}=-.02$ to .32 ), or from their scores at $180^{\circ}\left(p=.06, C I_{.95}=-.01\right.$ to .32$)$. The simple effects of angle were significant for nonsigners $[F(2,21)=$ $\left.14.16, p<.001, \eta_{\mathrm{p}}^{2}=.57\right]$ but not for signers $[F(2,21)=$ $\left.3.03, p=.07, \eta_{\mathrm{p}}^{2}=.22\right]$.

Consistent with the advantage found in Experiment 1, pairwise comparisons showed that signers and nonsigners differed significantly in the $180^{\circ}$ condition $(p=.04$, $C I_{95}=.01$ to .34 ) but not in the $0^{\circ}$ or $90^{\circ}$ conditions.

To ensure that a real difference between the groups in the less demanding $0^{\circ}$ condition was not being masked by ceiling effects, scores from longer sets at this orientation were also compared. For consistency with typical spatial span procedure, we compared proportion correct collapsed across sets of 3 to 7 items. The means (and $S D$ s) for signers and nonsigners were $.81(.15)$ and $.78(.06)$, respectively. An independent samples $t$ test confirmed no significant difference between signers and nonsigners in this condition $\left[t(22)=.55, p=.59, C I_{.95}=-.07\right.$ to .12].

The analyses indicate patterns of findings similar to those from Experiment 2, except that Experiment 3 did not find a significant interaction of group by angle. To assess whether the two input modes (manual tapping and remote controlled LEDs) affected performance differently, the data from both experiments were combined in a three factor ANOVA, with experiment as an additional two-level factor, to test for a higher level effect of experiment. This showed an overall group $\times$ angle interaction $\left[F(2,44)=4.63, p=.02, \eta_{\mathrm{p}}^{2}=.17\right]$, but no main effect of experiment $\left[F(1,22)=.17, p=.68, \eta_{\mathrm{p}}^{2}=.01\right]$, and no higher level interaction of experiment with any other factor $\left(F=.17\right.$ or lower; $p=.21$ or higher; $\eta_{\mathrm{p}}^{2}=.07$ or lower). This result suggests that the same overall pattern of findings is evident in Experiments 2 and 3.

\section{Discussion}

Experiment 3 showed that signers have an advantage for reproducing rotated spatial sequences even when there is no motor pattern at input. In contrast to nonsigners, they showed no significant cost of rotation, and furthermore their performance was significantly better than nonsigners when the arrays were rotated through $180^{\circ}$. Although Experiment 3 did not replicate the interaction of group $\times$ angle found in Experiment 2, a combined analysis confirmed that essentially the same pattern of findings was evident in both experiments. The resulting conclusion is that the signers' advantage for processing rotated arrays does not depend on seeing a motor pattern at input.

\section{GENERAL DISCUSSION}

The key result of the present investigation was that a group of hearing people who acquired sign language in adulthood outperformed hearing nonsigners on tasks involving immediate recall of rotated arrays. In Experiments 1 and 3, the signers scored significantly higher than the nonsigners on arrays rotated by $180^{\circ}$ in the horizontal plane, a spatial configuration that is consistent with canonical signing relations. This finding concurs with previous data from deaf native signers, who perform significantly better than nonsigners on tasks involving $180^{\circ}$ rotation (Emmorey et al., 1998). Experiments 2 and 3 showed that, in contrast to the nonsigners and to effects typically found in the literature (Shepard \& Cooper, 1986), the signers showed no significant costs associated with processing rotated arrays. This result is consistent with findings from native signers, who suffer no detriment to performance when presented with signed inputs requiring mental rotation (Emmorey et al., 1998). These novel results suggest that even a modest amount of experience with sign language, acquired in early adulthood, can produce measurable adaptations to cognitive functions in hearing people.

Returning to the key theoretical issue of which particular adaptations to working memory arise from sign language experience: What do the present data tell us? It is clear that some adaptation has taken place, as the signers have acquired a facility for processing rotated spatial arrays. By examining the processes that contribute to their advantage on these tasks, it may be possible to draw some conclusions about the cognitive mechanisms that have been affected by their linguistic experience. Prior to Experiment 3, we discussed two possible types of representation that seemed likely candidates for enhancement through experience of this linguistic form.

One account suggested that the signers might have utilized a motor representation of the experimenter's movements. As discussed earlier, evidence indicates that native signers represent sign movements internally, in a kinesthetic or motor code. If the present group of nonnative signers represented the spatial sequences using a motor code, this would suggest that signing experience affects mechanisms specialized for the patterning of physical actions. Studies have shown that a separable working memory subsystem is responsible for this type of function (Smyth, Pearson, \& Pendleton, 1988; Smyth \& Pendleton, 1989, 1990). The system deals with "movement that involves the patterning of action in time and space," and affords "the ability to watch another body move and to reproduce that movement with one's own body" (Smyth \& Pendleton, 1989, pp. 247-249).

The results of Experiment 3, however, showed that the signers did not depend on seeing a motor pattern at input, which does not suggest the operation of a configurational working memory system. Of course, we cannot rule out the possibility that they transformed the LED sequences 
into a motor code. The experiments were run in the order in which they are reported here, so that by Experiment 3 the participants had seen the sequences of blocks tapped manually on two previous occasions. To imagine a series of hand movements consistent with the LED sequences might therefore have been relatively easy, especially for the signers with their extended experience of processing such motor patterns. Our task may not be ideal for engaging configurational processes in any case (see Smyth \& Pendleton, 1989), so we cannot definitively conclude that no adaptation of motor representations occurs among nonnative signers. However, we have established that the signers' advantage in these studies was not critically dependent on seeing hand movements, and thus we cannot infer adaptations to configurational working memory from the present results.

The second account suggested that the underlying mechanism might be best conceptualized as a visuospatial function. In theoretical terms, this would implicate the visuospatial scratchpad or sketchpad component of working memory, described broadly by Baddeley (1997) as "a system assumed to be responsible for setting up and manipulating visuo-spatial images" (p. 71). A number of researchers have claimed that visuospatial working memory is fractionated into two separable subsystems (Logie, 1986, 1995; Logie \& Marchetti, 1991; Logie \& Pearson, 1997; Logie, Zucco, \& Baddeley, 1990). Separate resources or mechanisms have been proposed for the temporary storage or maintenance of visuospatial information on the one hand and, on the other, for the manipulation or transformation of such information.

Any attempt to account for the signers' advantage in terms of enhanced visuospatial storage capacity is undermined by the results of the Corsi blocks spatial-span test administered prior to Experiment 1. This measure indicated no difference between the groups in spatial STM capacity. It therefore seems more likely that the signers' advantage lay in their ability to manipulate or transform the spatial information, rather than in their ability to maintain it in STM.

An interesting question is what mechanism the signers employed during these tasks. If their advantage is best conceptualized in terms of enhanced rotational abilities, the underlying processes may simply be a more efficient version of those used by nonsigners. Previous research has shown that practice on mental rotation tasks can lead to faster rotation processes (Wallace \& Hofelich, 1992) and the ability among some individuals to manipulate an array as a gestalt rather than piecemeal (Bethell-Fox \& Shepard, 1988). It is possible that experience with sign language, in which spatial information is presented in varying orientations, produces similar effects. Alternatively, it is possible that the signers' advantage lay in spatial functions other than mental rotation per se. For example, signing experience may enhance the ability to encode viewpointinvariant or orientation-free representations of space (e.g., Biederman, 1987), or to rapidly generate multiple viewpoints (e.g., Tarr \& Pinker, 1989). Interestingly, Emmorey et al. (1998) argue that signers probably do not mentally rotate signing space during reception, citing the lack of a mental rotation effect and the intuitions of native signers, who report no sensation of rotating a mental image. They suggest instead that they may perform an instant reversal or repositioning transformation. Future studies examining response time profiles and eye fixation data could help to establish the cognitive basis for the signers' advantage and determine whether the underlying processes differ qualitatively or merely quantitatively from those used by nonsigners (Just \& Carpenter, 1976).

A related question is whether the signers' advantage should be characterized as a language effect; that is, whether it arises from sign language per se, or from general spatial processing demands. Would similar benefits transfer from nonlinguistic training containing a substantial spatial component or from expertise in nonlinguistic domains that involve related processes? The present data cannot answer this question, but if this effect is specific to sign language it might suggest that it is related to the representational code that signers use. Emmorey et al. (1998) raise the question of whether similar results from deaf native signers should be viewed as a "Whorfian effect." Although they conclude that such effects clearly demonstrate the influence of linguistic experience on cognitive systems beyond those used in communication, they also acknowledge that these phenomena are essentially complex practice effects arising from the repeated use of processes involved in language comprehension and production.

We interpret these results as arising from signing experience, but it is also possible that the signers were a spatially adept group even before they learned to sign. That is, hearing people who enter or succeed in sign interpreter training may self-select for spatial ability. To rule out the possibility of a self-selection bias, it would be necessary either to measure the spatial abilities of would-be interpreters before they embarked on any training, so that nonsigners could be matched on this variable; or participants might need to be randomly assigned to training and nontraining groups - although the latter would be a difficult design to implement, given the time necessary to acquire fluency in sign language. While we acknowledge the possibility of self-selection effects among interpreters, we would point out that enhancements to spatial processes have also been documented in native signers, both deaf and hearing, for whom there is clearly no likelihood of any preselection on the basis of spatial abilities.

In conclusion, our data are consistent with previous evidence from deaf native signers indicating that experience with sign language influences cognitive functions beyond the linguistic domain. A novel conclusion from the present study is that visuospatial working memory functions accrue adaptations even in hearing people who learn to sign in adulthood.

\section{AUTHOR NOTE}

We thank the anonymous reviewers for their detailed comments and suggestions, which resulted in a substantially improved theoretical discussion of the results. We also thank the BSL interpreters for participating in these studies. Correspondence concerning this article should be addressed to M. Keehner, School of Psychology, University of Dundee, Dundee DD1 4HN, Scotland (e-mail: m.m.keehner@dundee.ac.uk). 


\section{REFERENCES}

BADDeley, A. D. (1986). Working memory. Oxford: Oxford University Press.

BADDELEY, A. D. (1997). Human memory: Theory and practice (revised ed.). Hove, U.K.: Psychology Press.

BADDELEy, A. D., \& Hitch, G. J. (1974). Working memory. In G. Bower (Ed.), The psychology of learning and motivation: Advances in research and theory (Vol. 8, pp. 47-90). New York: Academic Press.

Baddeley, A. D., Thomson, N., \& Buchanan, M. (1975). Word length and the structure of short-term memory. Journal of Verbal Learning \& Verbal Behavior, 14, 575-589.

Bavelier, D., Corina, D. P., Jezzard, P., Padmanabhan, S., Clark, V. P., Karni, A., ET AL. (1997). Sentence reading: A functional MRI study at 4 Tesla. Journal of Cognitive Neuroscience, 9, 664-686.

Bethell-Fox, C. E., \& Shepard, R. N. (1988). Mental rotation: Effects of stimulus complexity and familiarity. Journal of Experimental Psychology: Human Perception \& Performance, 14, 12-23.

BiEDERMAN, I. (1987). Recognition-by-components: A theory of human image understanding. Psychological Review, 94, 115-147.

CAmpbell, R., \& DodD, B. (1980). Hearing by eye. Quarterly Journal of Experimental Psychology, 32, 85-99.

CoRINA, D. (1998). Studies of neural processing in deaf signers: Toward a neurocognitive model of language processing in the deaf. Journal of Deaf Studies \& Deaf Education, 3, 35-48.

Consi, P. M. (1973). Human memory and the medial temporal region of the brain. Dissertation Abstracts International, 34, 891.

Emmorey, K., Corina, D., \& Bellugi, U. (1995). Differential processing of topographic and referential functions of space. In K. Emmorey \& J. Reilly (Eds.), Language, gesture, and space (pp. 43-62). Hillsdale, NJ: Erlbaum.

Emmorey, K., Klima, E., \& HickoK, G. (1998). Mental rotation within linguistic and non-linguistic domains in users of American Sign Language. Cognition, 68, 221-246.

Emmorey, K., Kosslyn, S. M., \& Bellugi, U. (1993). Visual imagery and visual-spatial language: Enhanced imagery abilities in deaf and hearing ASL signers. Cognition, 46, 139-181.

Gardiner, J. M., Gathercole, S. E., \& GregG, V. H. (1983). Further evidence of interference between lipreading and auditory recency. Journal of Experimental Psychology: Learning, Memory, \& Cognition, 9, 328-333.

Hanley, J. R., Young, A. W., \& Pearson, N. A. (1991). Impairment of the visuo-spatial sketch pad. Quarterly Journal of Experimental Psychology, 43A, 101-125.

HickoK, G., Bellugi, U., \& KLima, E. S. (1996). The neurobiology of sign language and its implications for the neural basis of language. Nature, 381, 699-702.

Hitch, G. J., Halliday, M. S., Dodd, A., \& Littler, J. E. (1989). Development of rehearsal in short-term memory: Differences between pictorial and spoken stimuli. British Journal of Developmental Psychology, 7, 347-362.

Just, M. A., \& CARPEnter, P. A. (1976). Eye fixations and cognitive processes. Cognitive Psychology, 8, 441-480.

Klima, E., \& Bellugi, U. (1979). The signs of language. Cambridge, MA: Harvard University Press.

Klima, E., Tzeng, O., FoK, A., Bellugi, U., \& Corina, D. (1996). From sign to script: Effects of linguistic experience on perceptual categorization (Tech. Rep. No. INC-9604). University of California, San Diego: Institute for Neural Computation.

LogIE, R. H. (1986). Visuo-spatial processing in working memory. Quarterly Journal of Experimental Psychology, 38A, 229-247.

LogIE, R. H. (1995). Visuo-spatial working memory. Hove, U.K.: Erlbaum.

Logie, R. H., \& Marchetti, C. (1991). Visuo-spatial working mem- ory: Visual, spatial or central executive? In R. H. Logie \& M. Denis (Eds.), Mental images in human cognition (pp. 105-115). Amsterdam: North-Holland.

Logie, R. H., \& Pearson, D. G. (1997). The inner eye and the inner scribe of visuo-spatial working memory: Evidence from developmental fractionation. European Journal of Cognitive Psychology, 9, 241-257.

Logie, R. H., Zucco, G., \& BAdDELEy, A. D. (1990). Interference with visual short-term memory. Acta Psychologica, 75, 55-74.

Milner, B. (1971). Interhemispheric differences in the localization of psychological processes in man. British Medical Bulletin, 27, 272-277.

Neville, H., \& LaWson, D. (1987). Attention to central and peripheral visual space in a movement detection task: An event-related potential and behavioral study: II. Congenitally deaf adults. Brain Research, 405, 268-283.

PARASNIS, I., \& SAMAR, V. J. (1985). Parafoveal attention in congenitally deaf and hearing young adults. Brain \& Cognition, 4, 313-327.

Poizner, H., Bellugi, U., \& Tweney, R. D. (1981). Processing of formational, semantic, and iconic information in American Sign Language. Journal of Experimental Psychology: Human Perception \& Performance, 7, 1146-1159.

Poizner, H., FoK, A., \& Bellugi, U. (1989). The interplay between perception of language and perception of motion. Language Sciences, 11, 267-287.

Rettenbach, R., Diller, G., \& Sireteanu, R. (1999). Do deaf people see better? Texture segmentation and visual search compensate in adult but not in juvenile subjects. Journal of Cognitive Neuroscience, 11, 560-583.

RiCHARDSON, J. T. E., \& VECCHI, T. (2002). A jigsaw-puzzle imagery task for assessing active visuospatial processes in old and young people. Behavior Research Methods, Instruments, \& Computers, 34, 69-82.

SHEPARD, R. N., \& COOPER, L. A. (1986). Mental images and their transformations. Cambridge, MA: MIT Press.

Smyth, M. M., Pearson, N. A., \& Pendleton, L. R. (1988). Movement and working memory: Patterns and positions in space. Quarterly Journal of Experimental Psychology, 40A, 497-514.

Smyth, M. M., \& Pendleton, L. R. (1989). Working memory for movements. Quarterly Journal of Experimental Psychology, 41A, 235-250.

Smyth, M. M., \& Pendleton, L. R. (1990). Space and movement in working memory. Quarterly Journal of Experimental Psychology, 42A, 291-304.

SÖDERFELDT, B., RöNNBERG, J., \& RisBERG, J. (1994). Regional cerebral blood flow in sign language users. Brain \& Language, 46, 59-68.

TARR, M. J., \& Pinker, S. (1989). Mental rotation and orientationdependence in shape recognition. Cognitive Psychology, 21, 233-282.

Wallace, B., \& Hofelich, B. G. (1992). Process generalization and the prediction of performance on mental imagery tasks. Memory \& Cognition, 20, 695-704.

Wilson, M., Bettger, J. G., Niculae, I., \& Klima, E. S. (1997). Modality of language shapes working memory: Evidence from digit span and spatial span in ASL signers. Journal of Deaf Studies \& Deaf Education, 2, 150-160.

Wilson, M., \& Emmorey, K. (1997). A visuospatial "phonological loop" in working memory: Evidence from American Sign Language. Memory \& Cognition, 25, 313-320.

Wilson, M., \& Emmorey, K. (1998). A "word length effect" for sign language: Further evidence for the role of language in structuring working memory. Memory \& Cognition, 26, 584-590.

(Manuscript received October 9, 2005; revision accepted for publication February 8, 2006.) 